\title{
Targeted Spontaneous Reporting of Suspected Renal Toxicity in Patients Undergoing Highly Active Anti-Retroviral Therapy in Two Public Health Facilities in Uganda
}

\author{
Helen Ndagije • Victoria Nambasa $\cdot$ Elizabeth Namagala $\cdot$ \\ Huldah Nassali • Dan Kajungu • Gordon Sematiko • \\ Sten Olsson $\cdot$ Shanthi Pal
}

Published online: 7 March 2015

(C) The Author(s) 2015. This article is published with open access at Springerlink.com

\begin{abstract}
Background Although the national HIV control programme in Uganda has a well-established system for monitoring disease progression and treatment outcomes, monitoring of adverse drug reactions (ADRs) is inadequate. In order to address under-reporting of ADRs, the National Pharmacovigilance Centre, in collaboration with the HIV control programme, piloted a targeted spontaneous reporting (TSR) system as a complementary method to traditional spontaneous reporting.

Methods From April 2012 to March 2014, all cases of suspected renal toxicity in 10,225 patients on tenofovir-based regimens were monitored in the regional pharmacovigilance
\end{abstract}

Electronic supplementary material The online version of this article (doi:10.1007/s40264-015-0277-9) contains supplementary material, which is available to authorized users.

H. Ndagije ( $₫)$ · V. Nambasa · H. Nassali · G. Sematiko National Pharmacovigilance Centre, National Drug Authority, P.O. Box 23096, Kampala, Uganda

e-mail: hbyomire@nda.or.ug

E. Namagala

STD/AIDS Control Program, Ministry of Health,

Kampala, Uganda

D. Kajungu

Institute of Health and Society (IRSS), Universite Catholique de Louvain, Brussels, Belgium

S. Olsson

World Health Organization Collaborating Centre for

International Drug Monitoring, Uppsala, Sweden

S. Pal

Essential Medicines and Health Products Quality Assurance and Safety of Medicines World Health Organisation,

Geneva, Switzerland centres of Masaka and Mbale. The identification of renal toxicity was performed using serum creatinine, urinalysis, and other signs and symptoms of kidney injury.

Results There was one suspected renal toxicity reported for every 200 patients on a tenofovir-based regimen. Some of the serious reactions reported were death in two cases and bone demineralisation in five patients. Most of patients had been on treatment for 2 years. Those that had been on tenofovir for more than 4 years had raised serum creatinine levels, emphasising the importance of monitoring for the risk of renal damage for longer. We also found that the reporting rate of suspected ADRs for all medicines in the two sites increased almost fivefold during the implementation period.

\section{Key Points}

The targeted spontaneous reporting methodology can increase reporting of suspected adverse reactions to a drug.

Although the occurrence of renal toxicity associated with tenofovir is low, in a resource-limited setting where there is rapid scale-up of treatment and patients are initiated on a tenofovir regimen without initial screening, a monitoring programme for those that may develop renal toxicity should be put in place to encourage evidence-based decision making for improved patient safety.

Having adopted and leveraged on existing structures in the STD/AIDS Control Program, a platform for collaboration with the National Pharmacovigilance Centre was created, hence strengthening pharmacovigilance activities. 
Conclusion Although the occurrence of suspected tenofovir renal toxicity of HIV patients is low, there is need to monitor those at risk so as to prevent irreversible kidney injury. TSR can complement spontaneous reporting for collecting safety data on particular drugs and increase ADR reporting rates.

\section{Introduction}

Uganda is a resource-limited country with a high disease burden. Communicable diseases namely malaria, tuberculosis (TB) and HIV/AIDS account for $54 \%$ of the total burden of disease [1]. Access to essential medicines for treating common diseases such as malaria, pneumonia, $\mathrm{HIV}$, and diabetes mellitus at facility level is reported at 50-68 \% according to a survey conducted by the Ministry of Health in 2008 [2]. In order to address the high disease burden, the Ministry of Health is focused on rapidly expanding access to essential medicines through implementation of public health programmes and targeting the major communicable and non-communicable diseases. This increased access to medicines is expected to increase the number of people at risk of developing adverse drug reactions (ADRs).

The World Health Organization (WHO) Programme for International Drug Monitoring, recognises a reporting rate of over 200 suspected ADRs per million inhabitants/year, sent at least once a quarter from a national pharmacovigilance centre as good performance [3]. With more than 10 million Individual Case Safety Reports (ICSR) submitted from member countries of this WHO programme, VigiBase ${ }^{\circledR}$ is one of the sources of international safety data [4]. Analysis of the spontaneous reports in VigiBase ${ }^{\circledR}$ over a decade between the year 2000 and 2009 revealed that high-income countries had high reporting rates ranging from between 3 and 613 reports per million inhabitants per year while low-income countries had the lowest range at 0-21 [5]. Despite recognition of the occurrence and burden of ADRs and the need to report them, several studies show that there is widespread under-reporting of ADRs to spontaneous reporting systems, including serious or severe ADRs [6-9]. The 2014 reporting rate from Uganda in VigiBase $^{\circledR}$ corresponds to 20 reports per million inhabitants [10, 11]. A study conducted to assess pharmacovigilance systems in sub-Saharan Africa indicated that in Uganda, public health programmes do not routinely collect and share ADR data with the National Pharmacovigilance Centre (NPC) [12].

As of September 2013, the national STD/AIDS Control Program (ACP) had distributed antiretroviral (ARV) medicines to over 570,573 HIV-infected persons [13]. The
HIV/AIDS 2013 Progress Report [13] showed that cotrimoxazole was dispensed to over 800,000 persons living with HIV whether ARV active or naive. Although, this program has a well-established system for monitoring HIV prevalence, incidence, numbers on treatment and treatment outcomes, monitoring of ADRs due to ARVs is still inadequate.

In order to address under-reporting of ADRs due to ARVs, the NPC, in collaboration with the national ACP piloted the targeted spontaneous reporting (TSR) approach as a complementary method to the traditional spontaneous reporting method. TSR (sometimes called stimulated reporting) is a variant of spontaneous reporting that focuses on capturing ADRs in a well-defined group of patients on treatment [14]. The WHO developed this TSR methodology in 2010 in three countries including Uganda with support from the Monitoring Medicines Project under the seventh framework programme (FP-7) of the European Commission. Following a training workshop on this new methodology, Uganda adapted TSR to monitor renal toxicity among HIV patients on tenofovir-based regimens. TSR was the method chosen because it held the promise of a comprehensive monitoring method that was affordable and feasible in healthcare settings with limited human and financial resources. TSR was considered likely to build on the gains that had already been achieved by the existing efforts in promoting the role of pharmacovigilance as a best practice that improves the quality of care.

Although tenofovir had formerly been recommended as part of the alternative first-line ARV therapy (ART) regimen in the national guidelines and is now the preferred first-line regimen, little was known about its toxicity profile in the Ugandan population. Several studies on the use of tenofovir by patients with mild renal dysfunction or for longer durations indicated that it might be associated with renal toxicity [15-18]. In vitro studies also suggested a higher risk than has been observed in patients [19]. Patient populations are heterogeneous and treatment is complicated by the coadministration of tenofovir with other drugs that may enhance renal toxicity. Monitoring patients on a tenofovir-based regimen for a possible increased risk of renal dysfunction is therefore crucial. However, this is not always done due to a lack of laboratory facilities, inadequate knowledge for monitoring and work overload, especially in resource-limited settings. Failure to monitor patients to identify those who may be at risk of developing renal toxicity may result in significant deterioration in kidney function over a number of years as well as other complications.

The TSR method was adapted and piloted in the ACP among patients on tenofovir-based ART regimens seeking care from the two regional pharmacovigilance centres of Masaka and Mbale Regional Referral hospitals and their 
catchment health facilities. The purpose of this study was to pilot TSR using the example of renal toxicity to tenofovir in adults and to enhance pharmacovigilance within the ACP.

\section{Methodology}

\subsection{Implementation Design}

This was a pilot using TSR methodology to capture ADRs for HIV-positive clients who were initiated on tenofovirbased regimens. The Uganda health delivery system is comprised of the following levels:

- national referral

- regional referral

- district or general hospital

- health centre IV (Serves a county, should be able to admit patients having a senior medical officer and another doctor as well as a theatre for carrying out emergency operations)

- health centre III (Found in sub-county, runs an outpatient clinic, treating common diseases and offering antenatal care. Its led by a senior clinical officer and has a functioning laboratory)

- health centre II (serves a few thousand people, led by a registered nurse, runs an out-patient clinic treating common diseases and offering antenatal care)

- health centre I (structured as village health teams and working as a link between the community and the health facilities).

Health services are also provided in the private sector by for-profit health providers, not-for-profit healthcare facilities and traditional/complimentary medicine providers. As of June 2014, the Ministry of Health had accredited 1,572 public and private health facilities countrywide to offer ART services. The health workers involved in ART service delivery range from doctors, clinical officers, registered nurses and midwives to nursing assistants. The NPC has leveraged on the expertise in the national and regional referral hospitals and has designated these as regional pharmacovigilance centres. Two focal persons were appointed by the hospitals to coordinate activities in the region including the lower public health facilities.

\subsection{Site Selection}

From April 2012 to March 2014, all cases of suspected renal toxicity in patients on tenofovir-based regimens were monitored in the two regional pharmacovigilance centres of Masaka and Mbale. The population in the study sites had been estimated at about 20,000 HIV-positive patients on ART.

\subsection{Monitoring of the Tenofovir-Based Regimen}

For the pilot study, cases of suspected renal toxicity in patients on tenofovir-based regimens were targeted and monitored. Health workers in the pilot facilities were trained to monitor for signs and symptoms of tenofovir renal toxicity in all patients that were initiating or were already on tenofovir-based regimens. The laboratory tests carried out were serum creatinine and urinalysis (including proteinuria and glycosuria) as per the recommendations in the national guidelines for monitoring tenofovir at the various levels of health service delivery [20-22]. The 2014 national ART guidelines recommend carrying out urinalysis for monitoring of patients initiated on ART at baseline and thereafter every 6 months or as required [20]. They further recommend carrying out serum creatinine and/or blood urea to monitor renal function for patients on tenofovir every 6 months at district and regional referral hospitals.

The measurement parameters were the basis for the case definition and any patient that presented with any one outof-range parameter was considered for suspected renal toxicity. To simplify monitoring of patients at the pilot sites, a job aid was developed to guide health workers on the frequency of carrying out laboratory tests and on how to identify and report suspected cases. Signs and symptoms suggesting renal toxicity were described in the job aid (see Electronic Supplementary Material 1). Changes from the baseline parameters even in the absence of cardinal symptoms of renal insufficiency would trigger adverse reaction reporting.

\subsection{Training and Support Supervision for the Targeted Spontaneous Reporting (TSR) Methodology}

The health professionals managing patients were trained on the TSR methodology and renal toxicity monitoring as well as any other safety concerns. To supplement the training, support supervision was conducted regularly and involved personnel from both the NPC and the ACP. During support supervision, review meetings with the pharmacovigilance centre focal persons were held and strategies for strengthening pharmacovigilance in the hospitals were reviewed. Mentoring in ART care with focal facility personnel was also done.

\subsection{Data Collection}

Based on the case definition and the national guidelines, health workers reported suspected ADRs using the national 
form (see Electronic Supplementary Material 2). Completed reports were then relayed to the regional pharmacovigilance coordinator who entered them into the webbased case management system VigiFlow ${ }^{\mathrm{TM}}$ [23]. The NPC staff and the ACP focal person held regular meetings to assess causal relationships. The assessors assigned a score to the components and factors considered in establishing the causal relationships between tenofovirand the adverse events such as temporal sequence, alternative causes, confirmation by objective evidence and challenge and re-challenge with the drug. Each question was answered positive (yes), negative (no), or unknown or inapplicable (do not know).The likelihood of tenofovir being related to renal toxicity was assessed using the Naranjo algorithm [24]. The suspected ADR was then assigned to a category from the total score as follows: definite $\geq 9$, probable $5-8$, possible $1-4$, doubtful $\leq 0$. A descriptive summary of data on renal toxicity was created and is presented in Sect. 3.3.

\subsection{Ethical Considerations}

ADR monitoring and reporting is a routine clinical care activity. Health workers were required to report the reactions that they observe and those reported by the patients. Informed consent was not individually taken from each patient as the ADR form is confidential and was treated as such by the NPC. Ethical clearance was sought from the Uganda National Council for Science and Technology (Ref: HS 1136), which provides ethics research clearance

Table 1 Patients on the tenofovir-based regimen among the study sites

\begin{tabular}{lc}
\hline Study site & $\begin{array}{l}\text { Number of patients } \\
\text { on tenofovir }\end{array}$ \\
\hline Masaka Region & \\
Masaka Regional Referral hospital & 2,637 \\
TASO Masaka & 1,759 \\
Villa Maria hospital & 1,700 \\
Lyantonde hospital & 740 \\
Kalungu Health Centre IV & 63 \\
Kitovu hospital & 40 \\
Bukulula Health Centre IV & 348 \\
Subtotal & 7,287 \\
Mbale Region & \\
Mbale Regional Referral hospital & 950 \\
TASO Tororo & 1,615 \\
St. Antony hospital Tororo & 158 \\
Bududa hospital & 215 \\
Sub-total & 2,938 \\
Total & 10,225 \\
\hline
\end{tabular}

at the national level in accordance to the World Medical Association Helsinki Declaration. Approval for the pilot was obtained from National Drug Authority and the ACP to conduct this pilot study.

\section{Results}

\subsection{Background to the Sites}

A total of 10,225 patients were on tenofovir-based regimens in the two pilot sites in the 24 months of the pilot implementation. A majority of these patients were from the Masaka region as presented in Table 1.

\subsection{Demographics of the Suspected Renal Toxicity Cases}

Fifty-three $(0.52 \%$ of the exposed cases) of the suspected renal toxicities were reported to the NPC, of which 23 (43.4\%) were males. The median age of these patients was 43 years [interquartile range (IQR) 33-51] and the median body weight was $55 \mathrm{~kg}$ (IQR 47-62). Patients had been on tenofovir-based regimen for a median duration of 24 months (IQR 12-36). All patients that had been on a tenofovir regimen for more than 4 years had raised creatinine levels.

The majority ( $60 \%)$ of this cohort of patients were on the tenofovir/lamivudine/nevirapine (TDF/3TC/NVP) combination, while $35 \%$ were on tenofovir/lamivudine/efavirenz (TDF/3TC/EFV) and the remaining $5 \%$ were on the ritonavir-boosted lopinavir in combination with TDF/3TC.

\subsection{Presenting Signs and Symptoms for the Suspected Renal Toxicity}

Of the 53 cases with suspected renal toxicities, six (11\%) presented with bilateral pedal oedema, four with facial

Table 2 Summary of presenting signs and symptoms of suspected renal toxicity

\begin{tabular}{lll}
\hline Parameter & $\begin{array}{l}\text { Number of } \\
\text { patients }(\%)\end{array}$ & $\begin{array}{l}\text { Total } \\
(N)\end{array}$ \\
\hline Increased creatinine $(>1.2 \mathrm{~g} / \mathrm{dL})$ & $43(81)$ & 53 \\
Creatinine clearance $>90 \mathrm{~mL} / \mathrm{min}$ & $43(100)$ & 43 \\
Creatinine levels not provided & $10(19)$ & 53 \\
Proteinuria & $25(66)$ & 38 \\
Glycosuria and proteinuria & $11(44)$ & 25 \\
Bone demineralisation & $5(9)$ & 53 \\
Bilateral pitting pedal oedema & $6(11)$ & 53 \\
Facial puffiness & $4(8)$ & 53 \\
\hline
\end{tabular}


puffiness and five with bone demineralisation, which was confirmed by X-ray. Eleven (21\%) cases had out-of-range urinalysis, and the results are summarised in Table 2 along with other observed signs and symptoms of suspected renal toxicity. All of the patients that presented with glycosuria also had proteinuria. Two out of every three patients on a tenofovir-based regimen whose protein levels were tested $(n=38)$ had a positive result. Haematuria found in one patient out of the 53 reported cases was not expected. None of the patients in this study presented with hyperglycaemia.

Twenty-one patients were changed to a non-tenofovirbased regimen and their urine parameters and creatinine clearance improved thereafter. Tenofovir was substituted with zidovudine in six of these cases and the other 15 cases were substituted with abacavir. Two of those cases changed to abacavir had co-morbidities of both hypertension and diabetes, whereas two of the cases changed to zidovudine also had hypertension. A 45-year-old male, who had been on a tenofovir-based regimen for 5 years and presented with increased serum creatinine, glycosuria and proteinuria, was the only case with hypertension that was monitored without a regimen change as the signs and symptoms of renal damage resolved thereafter. Other co-morbidities reported included one case of lipodystrophy and one case of Kaposi's sarcoma. The laboratory findings for one case were consistent with Fanconi syndrome (i.e. hypouricaemia, hypokalaemia, generalised aminoaciduria and proteinuria).

About $80 \%$ of the cases tested had a raised serum creatinine level. A raised serum creatinine and calculated creatinine clearance of $<90 \mathrm{~mL} / \mathrm{min}$ (calculated with the Cockroft and Gault formula) was reported in all 43 patients that had a serum creatinine test performed and this is presented in detail in Table 3.

One in every five cases of suspected renal toxicity did not have a result for serum creatinine. The characteristics of the ten patients whose serum creatinine results could not be established are listed in Table 4. Six of the ten cases had their regimen changed, mainly because of bone demineralisation, and two patients died.

\subsection{Causality Assessment}

The majority $(80 \%)$ of the suspected renal toxicity cases were classified as probable and the rest were possibly related to tenofovir. None of the causality assessment grading belonged to the extreme categories of doubtful and definite.

\subsection{Reporting Trends for Suspected Adverse Drug Reactions}

During the time of the pilot, there was an increase in the overall number of ADR reports that VigiBase ${ }^{\circledR}$ submitted to the national pharmacovigilance system compared with the previous years (shown in Fig. 1) and also specifically from the annual number of reports collected in the two study sites of Mbale and Masaka (seen in Table 5 with comparisons before and after the TSR pilot period). The reports collected is about five times more than the pre-study period.

\section{Discussion}

\subsection{Background to the Study}

This pilot was implemented to establish the feasibility and positive effects that may accrue from a targeted spontaneous reporting methodology in resource-limited settings. In the ACP, the TSR was adapted to monitor the safety of patients on HIV treatment by focusing on suspected renal toxicity related to the use of tenofovir.

\subsection{Summary of Findings}

In our study, there was one suspected renal toxicity reported in every 200 patients on a tenofovir-based regimen. Among the serious reactions reported were the death of two patients and bone demineralisation, also known as osteomalacia, which occurred in five patients. Further assessment of the causality relationship revealed that these reports were probably or possibly but not definitely related to tenofovir. The median duration of treatment for the patients in this cohort was 2 years. However, all 13 $(100 \%)$ patients that had been on the tenofovir regimen for more than 4 years had raised creatinine levels. This group of patients emphasises the importance of monitoring for the risk of renal damage the longer patients are on tenofovir. We also found out that the reporting rate of suspected ADRs to all medicines in the two selected sites increased almost five-fold during the implementation of the TSR methodology.

\subsection{Suspected Adverse Reactions Associated with Tenofovir}

Tenofovir is a drug that is widely used for treatment of HIV infections in Uganda as more than half of the patients on ART in the two targeted study sites had it as part of their regimen [20].

Based on the case definition, the findings from this pilot support the presenting signs and symptoms of renal toxicity associated with tenofovir that are not different from that reported in the literature [16, 17]. According to the manufacturer, adverse reactions with a suspected relationship with tenofovir are classified as either uncommon $(\geq 1 /$ $1,000$ to $<1 / 100)$ or rare $(\geq 1 / 10,000$ to $<1 / 1,000)$ [25]. 


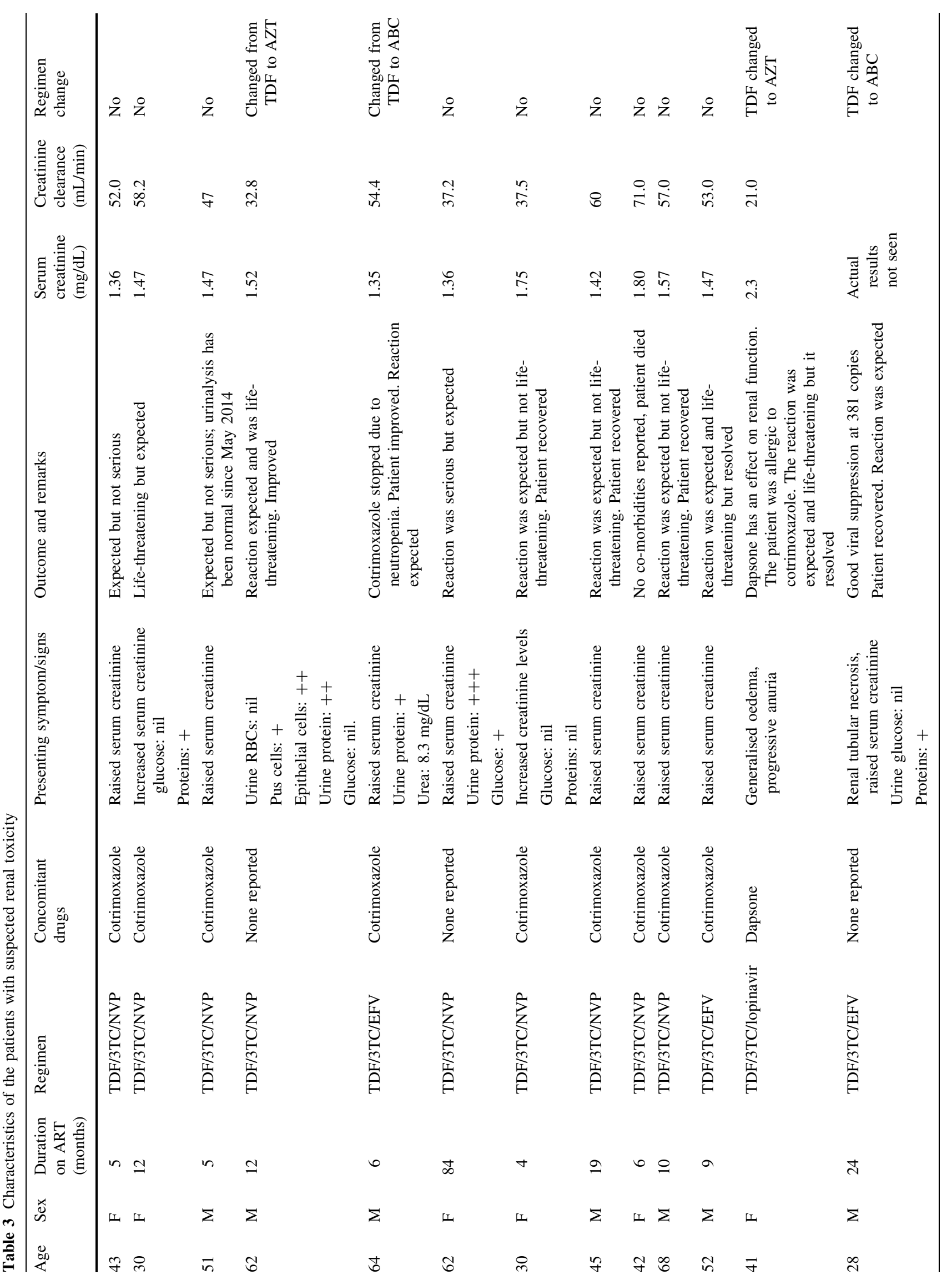




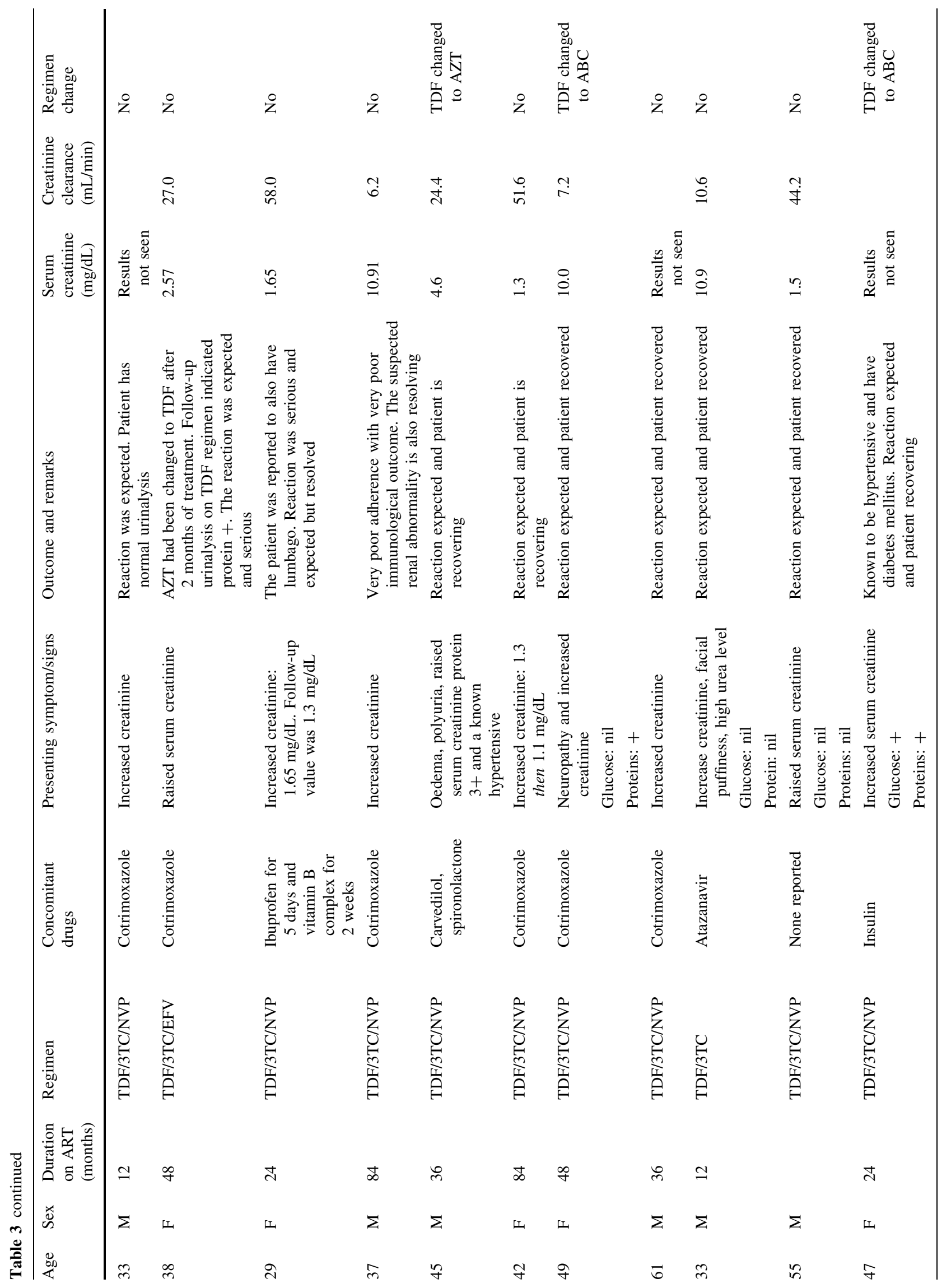




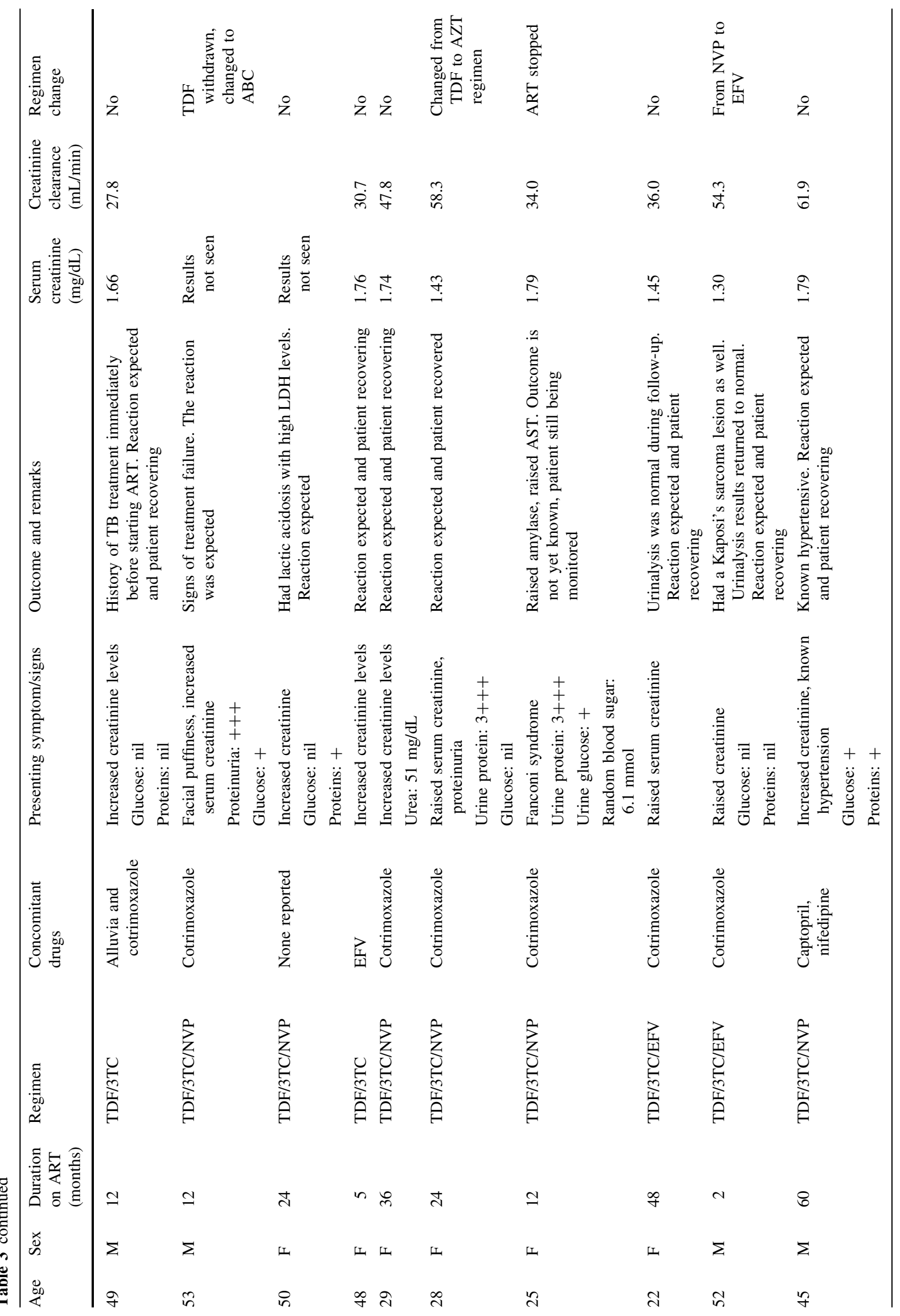




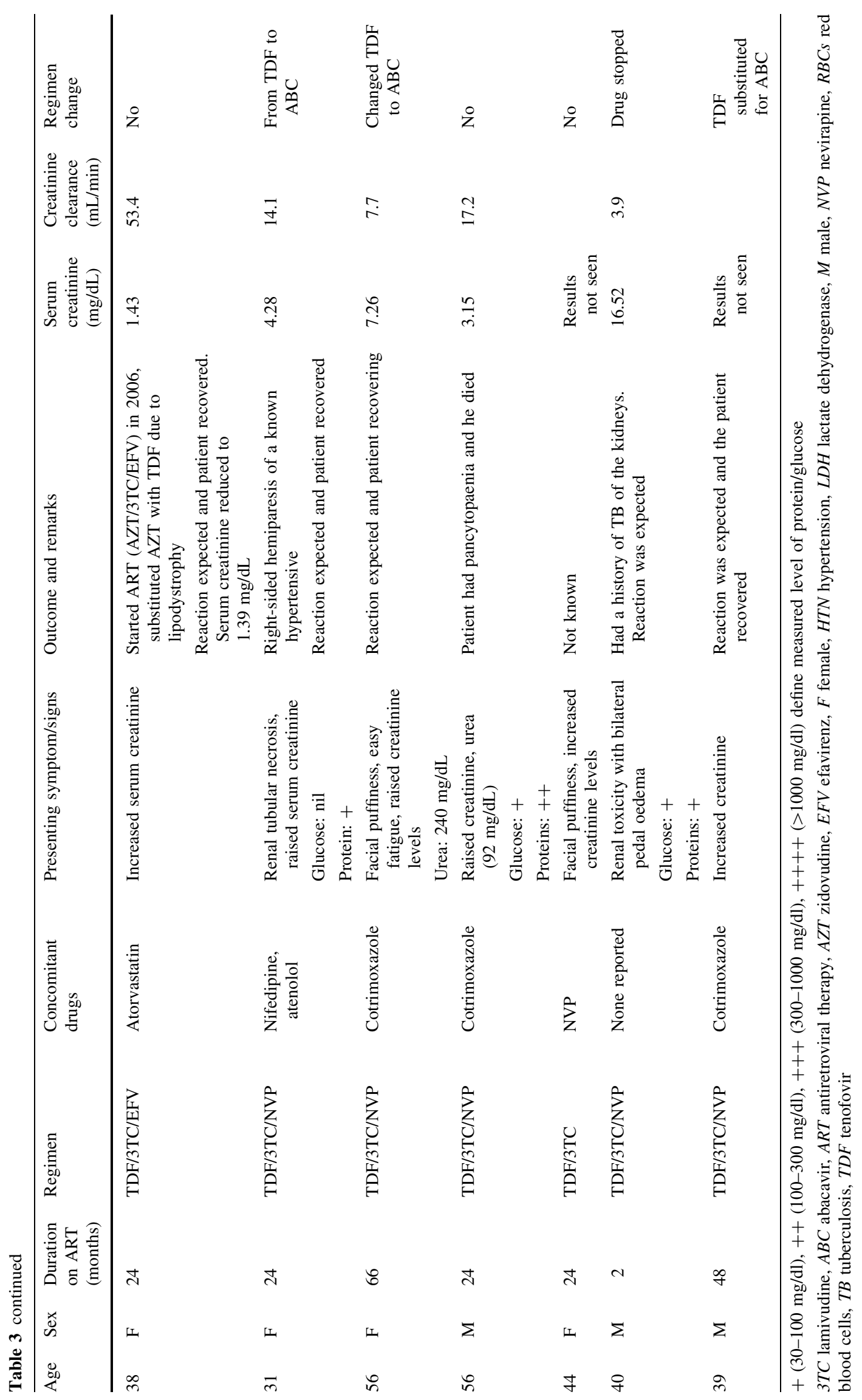




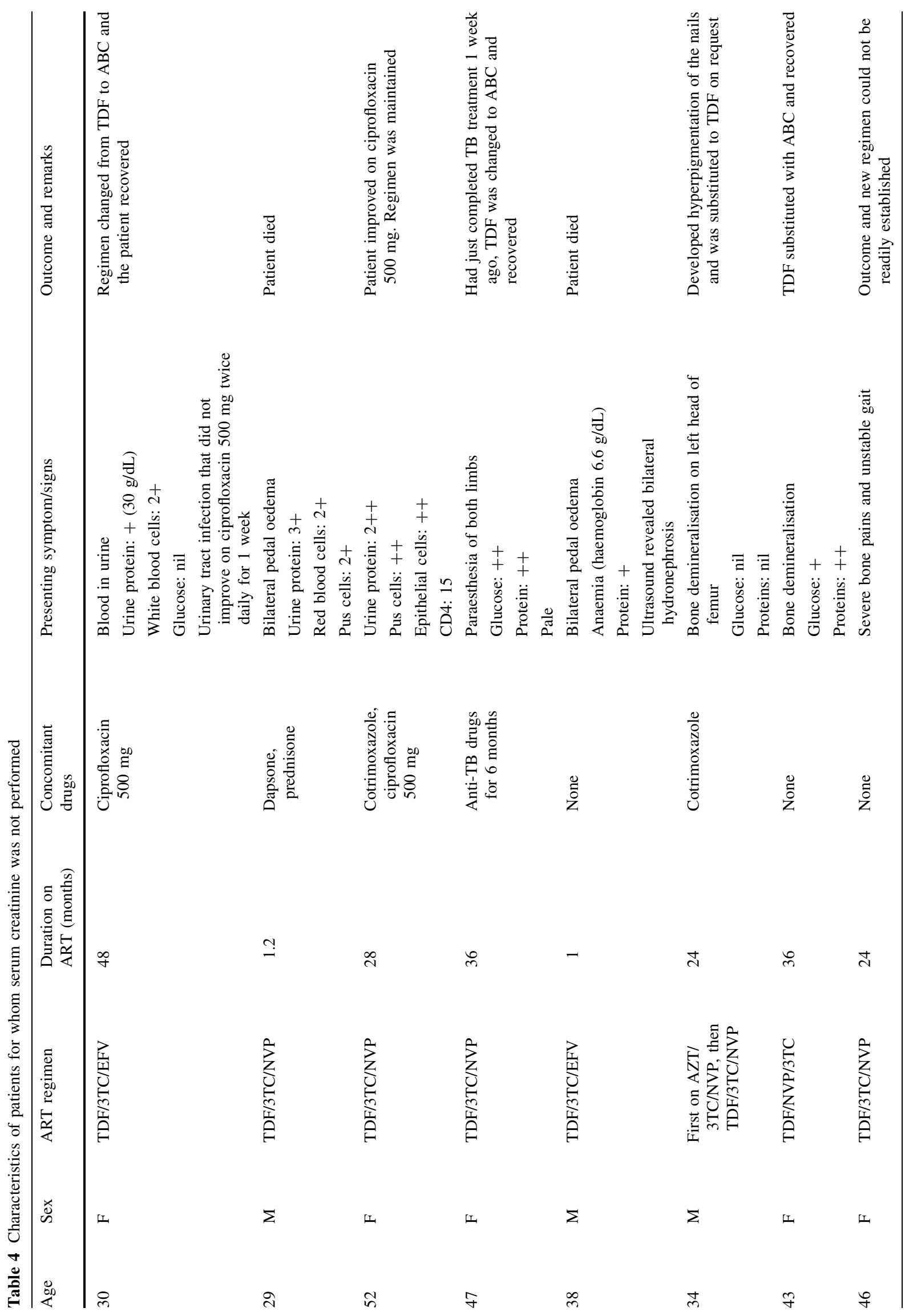


Increased serum creatinine was uncommon and renal failure, osteomalacia, lipodystrophy and angioedema were considered to be rare in the European Summary of Product Characteristics.

From our cases, proteinuria, glycosuria, raised serum creatinine and bone demineralisation are characteristics of tenofovir-induced kidney injury. Use of proteinuria and glycosuria to screen for tenofovir-induced kidney injury seems to be a very useful approach that should be emphasised and for which cost effectiveness ought to be studied further, especially for long-term monitoring of patients in resource-limited settings. Patients who had proteinuria diagnosed with dipstick, their regimens subsequently changed had a positive treatment outcome similar to other studies [16].

Since the main target for tenofovir toxicity is the proximal tubule, the presence of tubular proteinuria would be the most sensitive test for detecting proximal tubular dysfunction. However, it was not possible to measure tubular proteinuria in this pilot. The dipstick analysis used in this pilot primarily detected albuminuria, which is insensitive for low molecular weight proteinuria characteristic of tenofovir toxicity. The alternative tests for assessing proximal tubular function were to measure fractional excretion of phosphates and glycosuria, which are also established markers of proximal tubule dysfunction and are easy to screen for but are less sensitive tests than tubular protein excretion. Glycosuria was found in 11 of the patients, who also had proteinuria, while bone demineralization was found in five patients, which points to proximal tubular dysfunction $[16,25,26]$.

In studies [27, 28], serum creatinine $>1.2 \mathrm{~g} / \mathrm{dL}$ with calculated creatinine clearance $<90 \mathrm{~mL} / \mathrm{min}$ was reported in more than $80 \%$ of the cases. This is consistent with findings of a study of more than 1,000 HIV-infected patients on tenofovir which identified $1 \%$ whose serum creatinine increased to $>120 \mathrm{~g} / \mathrm{dL}$ [29]. The SCOLTA (Surveillance Cohort Long-Term Toxicity Antiretroviral) study of $754 \mathrm{HIV}$-infected, tenofovir-treated patients found a $2.5 \%$ incidence of creatinine elevations more than 1.5 times the upper limit of normal in a mean follow-up of 19.5 months, similar to the monitoring period of the patients in this pilot [29].

\subsection{Achievement of the TSR Pilot}

The project piloted TSR as a methodology for enhancing ADR reporting in the ACP in Uganda. It was specifically targeting patients who developed renal toxicity after taking tenofovir. This initiative was the first of its kind in Uganda and in sub-Saharan Africa.

The pilot was established on the basis of collaboration with a public health programme and the NPC, which 
Fig. 1 Number of individual case safety reports (ICSRs) from Uganda in VigiBase ${ }^{\circledR}$ per year

Table 5 Comparison of suspected adverse drug reaction reports collected from the study sites with the overall adverse drug reaction reports

TSR targeted spontaneous reporting

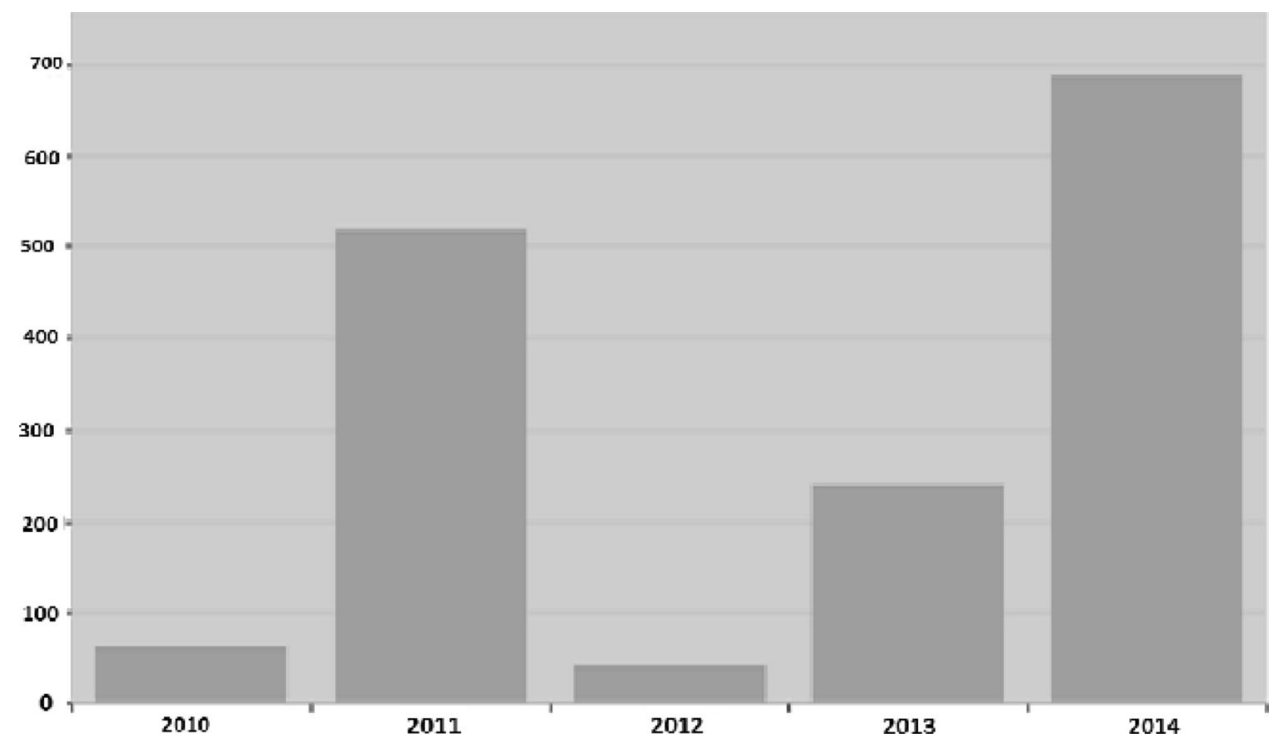

\begin{tabular}{|c|c|c|c|c|c|c|c|}
\hline \multirow[t]{3}{*}{ Source of reports } & \multicolumn{7}{|c|}{ Period } \\
\hline & \multicolumn{3}{|c|}{ Before TSR pilot study } & \multicolumn{4}{|c|}{ After start of TSR } \\
\hline & 2010 & 2011 & Total & 2012 & 2013 & 2014 & Total \\
\hline Study sites & 17 & 27 & 44 & 142 & 46 & 181 & 369 \\
\hline Uganda VigiBase ${ }^{\circledR}$ reports & 60 & 518 & 578 & 42 & 239 & 687 & 968 \\
\hline Percentage & & & $8 \%(44 / 578)$ & & & & $38 \%(369 / 968)$ \\
\hline
\end{tabular}

ensured ownership and division of activities according to the comparative advantage of each participating entity. The healthcare providers identified with the activity mostly because of the involvement of the ACP.

As a result of this collaboration, the ACP incorporated ADR reporting in its training manuals for ART. Also, pharmacovigilance reports from ART sites are incorporated into the national quarterly ART report. This has increased the number of spontaneous reports from public health facilities. It has increased visibility of the NPC activities by opening communication lines with all public health system programmes through sharing of reports, feedback meetings, case review meetings and joint training of healthcare providers.

The pilot was observational with no exclusion and inclusion criteria for those monitored. Patients were monitored in the routine clinical setting where risk factors and gaps in care were observed and studied. In the case of lifelong treatment such as ART, reporting lasts a lifetime as well [14]. The fivefold increase in the reporting rate compared to the pre-study period suggests that the TSR methodology addresses the problem of under-reporting that is seen with the traditional Ugandan spontaneous reporting.

Through joint support supervision activities, the NDA and NPC were able to identify gaps in health service provision. The point in case is the Ministry of Health ART guidelines which were not clear to health workers regarding monitoring patients on tenofovir. The NPC noted that health workers were opting for more expensive tests such as serum creatinine for monitoring tenofovir instead of a urine dipstick which is a cheaper alternative.

This pilot had a capacity-building component in terms of training, mentorship and feedback on the quality of care provided at the health facilities. This motivated health workers to provide better services and also to report suspected adverse reactions. At the pilot close-out dissemination meeting, stakeholders were particularly impressed by the feedback presented to them about the quality of reports as analysed by the NPC. Stakeholders requested that the TSR method of monitoring safety be rolled out to other parts of the country.

\subsection{Challenges and Limitations of the TSR Pilot}

HIV treatment is a rapidly evolving field whose guidelines keep changing. The recommendations for ART and preventing mother to child transmission (PMTCT) kept changing as the policies and guidelines changed. As a result, the planned TSR roll-out for PMTCT services has not yet been carried out. 
Assessment of the cases was difficult because some reports did not have baseline tests for renal function at the start of therapy. The dipstick analysis used in this pilot detected primarily albuminuria and is insensitive for low molecular weight proteinuria characteristic of tenofovir toxicity. Ideally, proteinuria should be assessed by formal measurement of the urine protein-to-creatinine ratio $(\mathrm{P}: \mathrm{C})$ or albumin-to-creatinine ratio $(\mathrm{A}: \mathrm{C})$. This was not possible in this study because the pilot used dipstick testing for detecting proteinuria and hence absolute urine protein values were not obtained. The lack of baseline laboratory tests in some reported cases makes it difficult to attribute the findings to tenofovir renal toxicity since it was not possible to rule out the existence of renal disease before tenofovir regimens had been started.

Monitoring for ADRs was initially viewed as an extra activity stretching the programme's logistics and resources. The NPC at one point provided some supplies and gave incentives to those involved in the project. Health workers viewed the programme as research that comes with added work since they already have a heavy workload from their usual work and so expected allowances. The joint support supervision encouraged the health workers and contributed to their change of attitude and the quality of patient care started improving.

\subsection{Recommendations}

Use of targeted surveillance monitoring strategies increases the number of reports and can help to identify risks that are associated with specific treatment in public health programmes. This plays an important role in signal detection. The role of co-morbidities such as hypertension and diabetes in this case also needs to be further studied as the TSR is rolled out. The numbers in this study were too small to come to a meaningful conclusion regarding their relationship to tenofovir-associated renal toxicity. The cost effectiveness of using these simple tests should also be further studied, especially in resource-limited settings.

Pharmacovigilance should be integrated into public health systems as a routine activity. However, there is still the need to plan for pharmacovigilance activities and resources such as having a focal person at the health facility level, especially where there are high patient loads, and a regional focal person to coordinate pharmacovigilance activities within the regions. In order to improve ADR reporting, the reporting of pharmacovigilance activities in Uganda needs to be simplified and harmonised with the current electronic reporting systems for the public health programmes and the health management information systems. Advocacy for integration should be enhanced by the relevant stakeholders and continuous information sharing. For successful implementation, involvement of different stakeholders is key because the issues encountered are better handled by a multi-disciplinary approach.

\section{Conclusion}

This pilot revealed that the incidence of suspected adverse events that are possibly related to the use of tenofovirbased regimens in HIV treatment is one report in 200 patients. We also found that the reporting rate of suspected ADRs to all medicines in the two selected sites increased almost fivefold during the implementation of the TSR methodology.

Targeted spontaneous reporting as a surveillance method can complement spontaneous reporting since it comes with the advantages of an increased reporting rate and the possibility of calculating the incidence of the ADR in question. In resource-limited settings, a successful monitoring programme for the safety of medicines used in public health programmes can be implemented and a specific question of interest can be answered in a specific population. This encourages use of evidence-based decision making for a better-quality patient care programme.

Acknowledgments The authors wish to thank all the staff of the National Pharmacovigilance Centre, especially Angela Bonabana and Christine Naluswata, for their work in data collection. Special thanks also goes to the doctors, nurses, pharmacists, pharmacy technicians, counsellors and all of the health workers at the ART and PMTCT clinics in the Masaka and Mbale Regional Referral Hospitals.

Funding and conflict of interest The study was supported in part with funds from the Research Directorate of the European Commission under the seventh framework programme (grant number 223566) and United Nations Innnovative Financing to Shape Markets for HIV/ AIDS, Malaria and Tuberculosis (UNITAID). Helen Ndagije, Victoria Nambasa, Elizabeth Namagala, Huldah Nassali, Dan Kajungu, Gordon Sematiko, Sten Olsson and Shanthi Pal have no conflicts of interest that are directly relevant to the content of this study.

Theme issue This article is part of a theme issue co-edited by Elliot G. Brown, Shanthi Pal and Sten Olsson. No external funding was used to support the publication of this theme issue.

Open Access This article is distributed under the terms of the Creative Commons Attribution Noncommercial License which permits any noncommercial use, distribution, and reproduction in any medium, provided the original author(s) and the source are credited.

\section{References}

1. Government of Uganda, Ministry of Health. 2010. Health sector strategic plan III 2010/11-2014/15. http://www.health.go.ug/docs/ HSSP_III_2010.pdf. Accessed 28 Oct 2014.

2. Government of Uganda, Ministry of Health, December 2008. Access to and use of medicines by households in Uganda: report 
of a survey conducted 2008. http://www.afro.who.int/en/ downloads/doc_download/5722-uganda-access-to-and-use-ofmedecines-by-households.html. Accessed 26 Dec 2014.

3. WHO Collaborating Centre for International Drug Monitoring, Reporting trends. Uppsala, Sweden. Updated 2014 Nov 25. http:// who-umc.org/graphics/28351.gif. Accessed 30 Dec 2014.

4. Lindquist M. VigiBase, the WHO global ICSR database system: basic facts. Drug Inf J. 2008;42(5):409-19.

5. Aagaard L, Strandell J, Melskens L, Petersen PSG, Hansen EH. Global patterns of adverse drug reactions over a decade: analyses of spontaneous reports to VigiBase ${ }^{\mathrm{TM}}$. Drug Saf. 2012;35(12): 1171-82.

6. Kiguba R, Karamagi C, Waako P, Ndagije HB, Bird SM. Recognition and reporting of suspected adverse drug reactions by surveyed healthcare professionals in Uganda: key determinants. BMJ Open. 2014;4(11):e005869.

7. Lazarou J, Pomeranz B, Corey PN. Incidence of adverse drug reactions in hospitalized patients: a meta-analysis of prospective studies. JAMA. 1998;279:1200-5.

8. Gurwitz JH, Field TS, Avorn J, McCormick D, Jain S, Eckler M, et al. Incidence and preventability of adverse drug events in nursing homes. Am J Med. 2000;109(2):87-94.

9. Hazell L, Shakir SA. Under reporting of adverse drug reactions: a systematic review. Drug Saf. 2006;29(5):385-96.

10. WHO Collaborating Centre for International Drug Monitoring, VigiLyze, Search and Analyse VigiBase, The WHO Global Database of Individual Case Safety Reports (ICSRs). Uppsala, Sweden. Updated 2014 Dec 11. https://vigilyze.who-umc.org. Accessed 30 Dec 2014.

11. Uganda Bureau of Statistics, 2014 Census Population [Internet]. Kampala; p. 6. Available from: http://unstats.un.org/unsd/ demographic/sources/census/2010_PHC/Uganda/UGA-2014-11.pdf. Accessed 26 Dec 2014.

12. Strengthening Pharmaceutical Systems (SPS) Program. Safety of medicine in sub-Saharan Africa: assessment of pharma-covigilance systems and their performance. Submitted to the US Agency for International Development by the Strengthening Pharmaceutical Systems (SPS) Program. Arlington: Management Sciences for Health; 2011. http://apps.who.int/medicinedocs/ documents/s19152en/s19152en.pdf. Accessed 26 Dec 2014.

13. Uganda STD/AIDS Control Programme. HIV and AIDS Uganda country progress report; 2013. Kampala; 2014. http://www. unaids.org/sites/default/files/country/documents/UGA_narrative_ report2014.pdf. Accessed 26 Dec 2014.

14. Pal SN, Duncombe C, Falzon D, Olsson S. WHO strategy for collecting safety data in public health programmes: complementing spontaneous reporting systems. Drug Saf. 2013;36(2):75-81.

15. Chua AC, Llorin RM, Lai K, Cavailler P, Law H. Renal safety of tenofovir containing antiretroviral regimen in a Singapore cohort. AIDS Res Ther. 2012;9(1):19.

16. Fernandez-Fernandez B, Montoya-Ferrer A, Sanz AB, SanchezNiño MD, Izquierdo MC, Poveda J, et al. Tenofovir nephrotoxicity: 2011 update. AIDS Res Treat. 2011;2011:354908.
17. Cooper RD, Wiebe N, Smith N, Keiser P, Naicker S, Tonelli M. Systematic review and meta-analysis: renal safety of tenofovir disoproxil fumarate in HIV-infected patients. Clin Infect Dis. 2010;51(5):496-505.

18. Mulenga LB, Kruse G, Lakhi S, Cantrell RA, Reid SE, Zulu I, et al. Baseline renal insufficiency and risk of death among HIVinfected adults on antiretroviral therapy in Lusaka, Zambia. AIDS. 2008;22(14):1821-7.

19. Vidal F, Domingo JC, Guallar J, Saumoy M, Cordobilla B, Sánchez De La Rosa R, et al. In vitro cytotoxicity and mitochondrial toxicity of tenofovir alone and in combination with other antiretrovirals in human renal proximal tubule cells. Antimicrob Agents Chemother. 2006;50(11):3824-32.

20. Ministry of Health, Uganda. The Integrated National Guidelines on Antiretroviral Therapy, Prevention of mother to child transmission of HIV and infant \& young child feeding. 1st ed. Kampala; 2011. http://www.emtct-iatt.org/wp-content/uploads/2014/ 05/GL_Integrated-National-Guidelines-on-ART-PMTCT-andIYCF-June-2011-MOH-Uganda_0.pdf. Accessed 16 Feb 2015.

21. Gupta SK. Tenofovir-associated Fanconi syndrome: review of the FDA adverse event reporting system. AIDS Patient Care STDS. 2008;22(2):99-103.

22. Romo FT, Aziz M, Livak B, Huesgen E, Colton B, et al. Renal function recovery and HIV viral suppression following tenofovir discontinuation for renal impairment. J AIDS Clin Res. 2014;5:379. doi:10.4172/2155-6113.1000379.

23. Olsson $\mathrm{S}$. The role of the WHO programme on international drug monitoring in coordinating worldwide drug safety efforts. Drug Saf. 1998;19(1):1-10.

24. Naranjo CA, Busto U, Sellers EM, Sandor P, Ruiz I, Roberts EA, et al. A method for estimating the probability of adverse drug reactions. Clin Pharmacol Ther. 1981;30(2):239-45.

25. Europeans Medicines Agency. Tenofovir summary of product characteristics. 2002. p. 1-183. http://www.ema.europa.eu/docs/ en_GB/document_library/EPAR_-_Product_Information/human/ 000419/WC500051737.pdf. Accessed 8 Jan 2015.

26. Hall AM, Hendry BM, Nitsch D, Connolly JO. Tenofovir-associated kidney toxicity in HIV-infected patients: a review of the evidence. Am J Kidney Dis. 2011;57(5):773-80.

27. Scherzer R, Estrella M, Li Y, Choi AI, Deeks SG, Grunfeld C, et al. Association of tenofovir exposure with kidney disease risk in HIV infection. AIDS. 2012;26(7):867-75.

28. Madeddu G, Bonfanti P, De Socio GV, Carradori S, Grosso C, Marconi $\mathrm{P}$, et al. Tenofovir renal safety in HIV-infected patients: results from the SCOLTA Project. Biomed Pharmacother. 2008;62(1):6-11.

29. Jones R, Stebbing J, Nelson M, Moyle G, Bower M, Mandalia S, et al. Renal dysfunction with tenofovir disoproxil fumarate-containing highly active antiretroviral therapy regimens is not observed more frequently: a cohort and case-control study. J Acquir Immune Defic Syndr. 2004;37(4):1489-95. 